\title{
Unscheduled return visits by patients to the accident and emergency department
}

F. O'DWYER \& G. G. BODIWALA

Department of Accident and Emergency Medicine, The Leicester Royal Infirmary, Leicester, England

\section{SUMMARY}

To determine why patients reattend an A\&E department we surveyed 235 patients who returned unscheduled in a one-month period.

Sixty-two per cent returned because of persistent symptoms. Sixty-three perc cent presented within a week of their initial visit. Only $32 \%$ had attempted to see $\frac{\mathbb{D}}{0}$ their GP. Thirty-five per cent of all patients claimed that A\&E staff had advized $\stackrel{\mathbb{D}}{3}$ them to return if they had problems. Half of the patients did not require treatment $\stackrel{\oplus}{\square}$ and $61 \%$ were discharged home. Twenty-one patients had pathology that hagl $\overrightarrow{0}$ been missed on their first visit. Better patient education may minimize misuse the service allowing better care for those who need it.

\section{INTRODUCTION}

Unscheduled return visits to an Accident and Emergency (A\&E) department present a considerable work-load. In the A\&E department of the Leicester Royal Infirmary these patients are seen by the registrar on duty. The aim of this study was to investigate unscheduled return visits to find out why patients returned, and if any conditions had been missed at the initial visit or the treatment altered.

\section{MATERIALS AND METHODS}

A pilot study was undertaken in the first week of February during which $\widetilde{\sigma}$ 50 patients were questioned. An improved questionnaire was devised and all

Correspondence: Frank O'Dwyer, Registrar in Accident and Emergency Medicine, The Leicester Royal Infirmary, Infirmary Close, Leicester LE1 5WW, England 
patients returning unscheduled during March were entered into the study. At the time of their return a questionnaire was filled in noting the time and day of their return, and the length of time since their last visit. The grade of doctor seeing the patient at both visits, the reason for the return and the source of referral were also noted. Where the patient was self referred, they were asked if they had seen their own doctor and why they had decided to return to A\&E with their problem. It was noted if the patient was X-rayed, what treatment was offered on their return and the outcome.

\section{RESULTS}

During March 8036 new patients were seen in the A\&E department of the Leicester Royal Infirmary. Of these $1078(13.4 \%)$ patients were reviewed in the department, 843 patients scheduled to come back to organized clinics and 235 patients as unscheduled 'return troubles' giving a total return rate of $2.9 \%$. These 235 patients form the study population. Of the 235 patients $147(63 \%)$ were male and $88(37 \%)$ were female. Twenty-one per cent were below sixteen years of age. A total of $39 \%$ were aged between 16 and 30 years. A total of $33 \%$ were aged between 31 and 65 years and $7 \%$ were aged over 65 years.

From Monday to Friday, $147(62.5 \%)$ of the 235 patients presented between 0800 and 1800 hours and $45(19.5 \%)$ presented after 1800 hours. Forty-two $(18 \%)$ presented on Saturdays or Sundays. There was little difference in the number seen on the various week days with fewer patients seen at week-ends.

A total of $149(63 \%)$ attended again within one week of their initial attendance, $58(25 \%)$ attended after a week to a month, and $28(12 \%)$ attended a month or longer after their initial visit.

On the initial visit, $141(81 \%)$ were seen by a senior house officer with the remaining $19 \%$ by a registrar, senior registrar or consultant. At the unscheduled return visit $212(90 \%)$ were seen by the registrar on duty, $7 \%$ by a senior registrar or consultant and $3 \%$ by a senior house officer.

The reason for the return was persistence in symptoms in 145 cases $(62 \%)$ and 29 $(12.5 \%)$ were due to a complication of treatment. A total of $31(13 \%)$ patients thought that 'something might have been missed'. The remaining $29(12.5 \%)$ gave a variety of reasons, such as initially had been seen and treated in the A\&E department'.

Of the 235 patients, $171(73 \%)$ had referred themselves back to the A\&E department. Fifty-three (23\%) were referred back by their GP, of whom $35(15 \%)$ arrived with a letter. The remaining 18 patients $(8 \%)$ said that they had seen their GP who advised them to reattend the A\&E department. Four per cent were referred by other sources e.g. the first-aider or occupational doctor/nurse at work. Amongst the 171 self referred patients, $150(86 \%)$ had made no attempt to see their GP, $14(9 \%)$ had seen their GP but were not specifically advised to return to the A\&E department and the remaining 7 (5\%) had attempted to see their GP but had been unable to do so. Among the reasons for reattending A\&E among self-referred 
patients, $48 \%$ of patients claimed that they were asked to come back by A\&E staff if required (see Table 1)

Forty-three per cent of patients were $X$-rayed at their initial visit only. Twelve $\frac{3}{0}$ per cent were $X$-rayed on their subsequent visit only, $15 \%$ were $X$-rayed on both? occasions and $30 \%$ were not $X$-rayed on either visit. Of the $27(12 \%)$ patients who were only $X$-rayed on their return, there were 6 positive findings.

Of the $34(15 \%)$ patients $X$-rayed on both occasions there were 5 previously unsuspected fractures.

A total of 11 patients had fractures that were not suspected at their initial ${ }_{0}^{\circ}$ attendance and one patient had a metacarpal dislocation (this patient did not wait ${ }^{\circ}$ for an X-ray). Five patients returned with serious infections that required treatment. One patient who presented initially with shoulder pain returned three days later $\vec{\omega}$ with a pneumothorax. The remaining patients had a variety of problems (see $\frac{\sigma}{\Phi}$ Table 2).

Seven patients returned with problems where they had refused to wait for treatment initially.

Table 1. Reasons for reattending A\&E.

\begin{tabular}{lr}
\hline Told to return by A \& E doctor if problems. & $34 \%$ \\
Told to return by A \& E nurse if problems. & $14 \%$ \\
More convenient to visit the hospital. & $18 \%$ \\
GP would refer anyway. & $13 \%$ \\
GP not available. & $10 \%$ \\
To save time. & $4 \%$ \\
Technical reasons (e.g. X-rays.) & $4 \%$ \\
Seen initially in A \& E. & $3 \%$ \\
\hline
\end{tabular}

Table 2. Missed pathology

\begin{tabular}{ll}
\hline Metacarpal fractures & 4 \\
Wrist fractures & 2, one greenstick \\
Trapezium fracture & 1 \\
Thumb proximal phalanx fracture & 1 \\
Osteochondral fracture dome of talus & 1 \\
Rib fractures & 1 \\
Zygoma fracture & 1 \\
Dislocated index metacarpo-phalangeal joint & 1 \\
Spontaneous Pneumothorax & 1 \\
Flexor sheath infection of thumb & 1 \\
Pulp space infection of finger & 3 \\
Paronychia & 1 \\
Missed Digital nerve injury & 1 \\
Perforated ear-drum & 1 \\
Foreign Body in eye & 1 \\
\hline
\end{tabular}




\section{Treatment}

Of the total, $50 \%$ of patients were offered advice only, $18 \%$ had a dressing applied, $8 \%$ were given a prescription (usually for an antibiotic or a non-steroidal anti-inflammatory drug) and $5 \%$ were given a splint or put in a plaster cast. Seven per cent of patients underwent surgery, $6 \%$ were referred for physiotherapy and the remaining $6 \%$ had other forms of treatment (e.g. steroid injection for frozen shoulder or tenosynovitis).

Surgery involved incision and drainage of an abscess in nine cases, draining pulp-space infections in two, flexor sheath infections in two, one digital nerve repair and one MUA of a dislocated MCP joint of a hand. On reviewing the notes the initial treatment was thought to be inappropriate in $8 \%$ of cases.

\section{DISCUSSION}

In all, 39\% of patients who returned unscheduled required further follow up suggesting that they were correct in re-attending the A\&E department. This is similar to patients who refer themselves to the A\&E department having first seen their General Practitioner (Jones et al., 1989). Access to the department should not be denied to these patients but many do not need immediate attention and can be advised to return to an organized review clinic.

In Leicester computerized letters are sent to all GPs following patient attendance at the A\&E department. Nearly all the GPs and read and file these letters and the majority find them helpful. (DaCruz et al., 1989) Despite this we have found few patients who try to contact their GP before re-attending. Only a third had seen or attempted to see their GP. Few patients in fact attempt to see their GP before attending A\&E departments at any time (Lewis, 1988, Lewis, 1981). Lermans et al. (1987) studied return visits to an Emergency department in Michigan and concluded that $15 \%$ of reattendances could have been avoided by better patient education. This may be applicable to a number in our study group.

A large number of these patients could have been dealt with equally effectively by their GP. A number of authors have addressed the problem of inappropriate attendances at the A\&E department (Driscoll et al., 1987). Meyers (1982) found that $47 \%$ felt that their GP could not provide treatment but he felt that $54 \%$ could have been treated by their GP, while Singh (1988) believed that patients perceptions of their problems and the availability of their GP were the main reasons for attending A\&E departments. The same factors are important in patients decisions to reattend the A\&E department. Patients need to be educated to accept their GP can look after most problems they may have after their visit to the A\&E department.

Getting the registrar on duty to see the 'return troubles' is justified. Nine per cent of patients had conditions missed on their initial visit and care had been inappropriate in $8 \%$ of cases. 


\section{REFERENCES}

DaCruz D. J., Austin S., Richardson S. \& Bodiwala G. G. (1989) The Value of computerized discharge $\frac{乛}{\mathbb{2}}$ letters. British Journal of Accident and Emergency Medicine 4 (2), 6-9.

Driscoll P. A., Vincent C. A. \& Wilkinson M. (1987) The use of the Accident and Emergency Department. $\stackrel{\overline{\bar{S}}}{\vec{F}}$ Archives of Emergency Medicine 4, 77-82.

Jones C. S. \& McGowan A. (1989) Self referral to an Accident and Emergency Department for another opinion. British Medical Journal 298, 859-62.

Lerman B. \& Kobernick M. (1987) Return Visits to the Emergency Department. Journal of Emergency Medicine 5, 359-62.

Lewis B. \& Bradbury Y. (1981) Why patients choose A\&E. Health and Social Services Journal, 1139-42.

Lewis H. (1987) Accident and Emergency department utilization: a consumer survey. New Zealand $\vec{O}$ Medical Journal 4, 77-82.

Meyers P. (1982) Management of minor problems and trauma: general practice or hospital. Journal of $\vec{\omega}$ the Royal Society of Medicine 75, 879-93.

Singh S. (1988) Self referral to Accident and Emergency Departments: Patients perceptions. British 3 Medical Journal 297, 1179-80. 